\title{
Not Meeting Criteria
}

National Cancer Institute

\section{Source}

National Cancer Institute. Not Meeting Criteria. NCI Thesaurus. Code C110964.

Indicates that an individual fails to meet the criteria for a particular study. 